\title{
Costimulatory Molecules CD80 and CD86 Colocalized in Neutrophil Extracellular Traps (NETs)
}

\author{
Rodriguez FM and Novak ITC* \\ Institute of Cell Biology, Faculty of Medical Sciences, National University of Cordoba, Argentina
}

${ }^{*}$ Corresponding author: Novak ITC, Enrique Barrios corner Gordillo. Ciudad Universitaria, (5000) Cordoba, Argentina, Tel: 0054351433 4020,E-mail: inovak@cmefcm.uncor.edu

Citation: Rodriguez FM, Novak ITC (2016) Costimulatory Molecules CD80 and CD86 Colocalized in Neutrophil Extracellular Traps (NETs). J Immunol Infect Dis 3(1): 103

Received Date: March 19, 2016 Accepted Date: April 26, 2016 Published Date: April 27, 2016

\begin{abstract}
Neutrophil extracellular traps (NETs) have been proposed as vehicles for the sensitization of T cells by decreasing their activation threshold. Polymorphonuclear neutrophils (PMNs) activated in vitro may express molecules normally associated with antigen presenting cells (APCs). May NETs contain costimulatory molecules? We perform the generation of NETs in autologous leukocytes cultures, stimulated with lipopolysaccharide (LPS) or ovalbumin (OVA). We marked of B7-1(CD80) and B7-2(CD86) costimulatory molecules. In LPS and OVA assays CD80 was found colocalized with CD86 in the released NETs at 30 min. In LPS assay at 120 min CD80 and CD86 colocalized with neutrophil elastase (NE) in NETs. These costimulatory molecules were detected on the cell surface at $30 \mathrm{~min}$ and $24 \mathrm{~h}$ but not in the control paired blood samples. In OVA assays fewer NETs were observed. We generated Monocyte-macrophage Lymphocytic Rosettes (MLRs) with OVA stimulation. A higher number of MLRs was formed in samples with presence of CD80 and CD86 in NETs. The expression of costimulatory molecules in NETs would allow PMNs to exert function as APCs and modulatory functions of various subpopulations of T cells. The presence of CD80 and CD86 in NETs could influence the cell environment through the B7-1/B7-2:CD28/CTLA-4 pathway. This is the first communication of CD80 and CD86 costimulatory molecules in NETs.
\end{abstract}

Keywords: CD80; CD86; NETs; Human neutrophils; Costimulatory molecules

\section{Introduction}

While polymorphonuclear (PMN) neutrophils play a crucial role in protecting against infection, recent evidence suggests that participation plays a key role in innate and adaptive responses mechanisms as effectors and regulators[1,2]. In inflammatory conditions, PMN neutrophils can release extracellular traps (NETs) [3,4] composed of chromatin, histone and granule proteins, which trap and kill a variety of microbes. Immunity has been proposed as second function of chromatin released in NETs [5]. The formation of NETs with sterile inflammatory stimuli from crystals of monosodium urate (MSU) has been of great importance in patients with gout [6]. Neutrophil extracellular traps (NETs) have been proposed as vehicles for the sensitization of T cells by decreasing their activation threshold, linking innate and adaptive immune responses [7]. NETs have also been implicated in autoimmunity, thrombosis and tissue injury [8] and in cancer immunoediting [9].

The mechanism of NET formation implicate morphological changes of PMN: after activation, the cells flatten and they attach to the substratum, the nucleus loses the lobules and the chromatin decondenses Nuclear membranes progressively lose their integrity. At the same time, granules desintegrate. Elastase migrates from the azurophilic granules to the nucleus and partially degrades histones promoting chromatin decondensation, myeloperoxidase synergizes this action [10]. After 1 hour, the components of nucleus and cytoplasm mix. Finally, the plasmatic membrane ruptures and ejects the interior of the cell to the extracellular space forming NETs [5]. Two mechanisms for the release of NETs in response to various stimuli are described: 1. early, quick release (5 to 60 minutes after in vitro stimulation with Staphylococcus aureus or lipopolysaccharide (LPS) and 2. slow (2 to 4 hours) leading to the death of the PMN "NETosis" [11]. Some authors summarize the release of NETs as "vital NETosis" in the case of PMN which are still alive, or as "lytic suicide NETosis" when drifting in the death of PMN [12,13].

PMNs activated in vitro may express molecules normally associated with antigen presenting cells required for T cell activation. B7 molecules (B7-1/CD80 and B7-2/CD86) are integral membrane glycoproteins that are expressed on the surface of APCs. CD86 exhibits constitutive expression on resting monocytes, macrophages and dendritic cells. In the same cells without stimulation CD80 expression is absent or low [14]. Engagement neutrophil CD11b receptor results in rapid surface expression of molecules required for antigen presentation and T cell activation, such as MHCII HLA-DR, CD80 and CD86. These costimulatory molecules are pre-formed in cytoplasmic reservoirs and are translocated to the surface with the engagement of certain receptors as MAC-1 
$(\mathrm{CD} 18+\mathrm{CD} 11 \mathrm{~b})[15]$. T cell activation initiates with a first signal, the interaction of the antigenic peptide/major histocompatibility complex with the TCR, and a second signal, the "costimulatory signal". The absence of costimulation in naive T cells results in antigen-specific unresponsiveness [16,17]. The second signal can be stimulatory or inhibitory, this depends on the receptor [17,18]. The B7-1/B7-2:CD28/CTLA-4 pathway is the best characterized T-cell costimulatory pathway [19,20]. CD28 is constitutively expressed on naive T cells; in contrast, expression of CTLA-4 is induced upon T cell activation. Activated T cells as well as regulatory $\mathrm{T}$ cells (Treg) with a pivotal role in the maintenance of peripheral self tolerance, express CTLA-4 [21]. The inhibitory receptor CTLA-4 (CD152) was found to be a higher affinity binding for CD80 and CD86, as compared to CD28 [22]. Transendocytosis of CD80 and CD86 by CTLA4 has been described. CTLA-4 captures ligands (CD80 and CD86) from APC and degrades them inside the CTLA-4-expressing cell. This process removes B7 molecules from neighboring cells and these results in impaired costimulation via CD28 [21,23].

In this study we have investigated the possibility of localization of costimulatory molecules in NETs. In the present work we perform assays to generate NETs in autologous leukocytes cultures, challenged with LPS and OVA and mark CD80 and CD86 costimulatory molecules. We have decided to use LPS; because it is one of the most recognized PMN activators, and OVA because in other granulocytes it induces formation of extracellular traps [24] and we have investigated the possibility of generating NETs. An experiment with $\mathrm{T}$ cells was performed in order to assess potential functional relevance of CD80 and CD86 colocalized in NETs. We generated immunological synapses in Monocyte-macrophage Lymphocytic Rosettes (MLRs) [25-28] with addition of OVA (heterologous antigen) in total autologous leukocyte cultures. It has been described that lymphocytes forming MLRs are T-CD4 cells [26]. In a previous study we showed that addition of heterologous antigen stimulated MLR phenomenon in cultures of purified mononuclear cells [28].

\section{Materials and Methods}

\section{Blood samples}

Heparinized human blood samples $(n=10)$ were collected with ethical consent according to procedures approved by ethical committee of National Hospital Clinicas R169/13, I minute book N129. Samples donated by the Blood Bank, Institute of Hematology and Hemotherapy of the National University of Cordoba in anonymity, with negative serology: Hudleson (Wiener), VDRL (Wiener), Chagas HAI (Wiener) Chagas EIE (Biomerieux), HBs EIE (Biomerieux), HBc (Biomerieux), HCV EIE (Murex), HIV Ac EIE (Biomerieux), HIV Ag EIE (Biomerieux), HTLV EIE (Murex).

\section{Autologous cell cultures}

Blood samples were allowed to sediment for 2 hours. Plasma supernatants containing all types of leukocytes were separated and were cultured at $37{ }^{\circ} \mathrm{C}$ in TC199 medium (Sigma, St-Louis, MO) supplemented with L-glutamine (Sigma, St. Louis, MO), added with filtered serum from the same donor. A 24-well cell culture plate was prepared by putting a sterile $13 \mathrm{~mm}$ round glass cover slip into each well.

\section{NET formation}

Cultured cells were stimulated with LPS to form NETs. (Lipopolysaccharides from Escherichia coli, Sigma-Aldrich) $25 \mathrm{ng} / \mathrm{ml}$ or OVA $100 \mu \mathrm{g} / \mathrm{ml}$ (Ovalbumin, Difco, Detroit, Michigan) at $37^{\circ} \mathrm{C}$ in a $\mathrm{CO}_{2}$ incubator. Samples: 30 minutes, some experiments at 120 minutes and 24 hours. The released NETs were visualized by fluorescence microscopy using DAPI (4,6'-diamidino-2-phenylindole) (Sigma, St Louis, MO) and anti-Neutrophil Elastase (anti-NE, Abcam).

\section{NETs quantification}

The released NETs were visualized by immunofluorescence microscopy and the percentage of cells releasing NETs and the percentage of cells with CD80 and CD86 colocalized in NETs was calculated as the average of six fields (400x) normalized to the total number of cells.

\section{Samples for (IF) Immunofluorescence}

Glass cover slips with attached cells were carefully removed from culture plate and IF techniques were performed.

\section{NE immunofluorescence staining}

Cultured cells after stimulation with LPS for 30 and 120 minutes washed briefly in PBS (phosphate buffered saline), fixation was performed with $4 \%$ paraformaldehyde for 10 minutes and washed in three changes in PBS. It was permeabilized in $0.25 \%$ Triton $\mathrm{x}-100$ in PBS. It was incubated with 5\% blocking serum albumin in PBS to prevent non-specific staining for 20 minutes. It was washed with PBS. It was incubated with a primary antibody anti-NE (rabbit Abcam) for one hour at $37^{\circ} \mathrm{C}$, washed in PBS and further incubated for one hour at $37^{\circ} \mathrm{C}$ with goat anti-rabbit IgG secondary antibody Alexa Fluor 594 conjugated or with goat antirabbit IgG-FITC fluorescein isothiocyanate (Santa Cruz Biotechnology). It was washed with PBS and nuclear staining with DAPI (4,6'-diamidino-2- phenylindole) (Sigma, St Louis, MO). It was mounted with medium 90\% glycerol in PBS. In all experiments, secondary antibody alone served as a negative control. Observation of preparations were performed in videomicroscope Axioscop 20, MC80, trinocular, Carl Zeiss. Paired blood samples provided controls. 


\section{CD80 and CD86 immunofluorescence staining}

After stimulation with LPS or OVA, culture cells washed briefly in PBS (phosphate buffered saline), fixation was performed with $4 \%$ paraformaldehyde for 10 minutes and washed in three changes in PBS. It was incubated with 5\% blocking serum albumin in PBS to prevent non-specific staining for 20 minutes. It was washed with PBS. It was incubated with antibodies (Ab) Santa Cruz Biotechnology anti-CD80 (FITC; Santa Cruz Biotechnology), anti-CD86 (PE; Santa Cruz Biotechnology) at $4{ }^{\circ} \mathrm{C}$ overnight. It was washed with PBS and nuclear staining with DAPI (4,6'-diamidino-2- phenylindole) (Sigma, St Louis, MO). It was mounted with mounting medium 90\% glycerol in PBS. Observation of preparations was done in videomicroscope Axioscop 20, MC80, trinocular, Carl Zeiss. Paired blood samples provided controls. We performed IF to CD80 and CD86 in samples from MLRs assay, see next section.

\section{MLRs Assay with OVA stimulation}

Cultured total autologous leukocytes were stimulated with OVA. Samples of these cultures were harvested at 24 hours and centrifuged at $200 \mathrm{~g}$ and cytopreparations were made of each cell pellet by cytospin process at $82 \mathrm{~g}$ (Thermo Scientific Cytospin 4). Control experiments were leukocyte cultures of the same donors without OVA. We assessed the formation of rosettes between blood monocyte-macrophages and lymphocytes (MLRs) in cultures [25-27]. Cytopreparations from each experiment were stained with May Grünwald Giemsa or Toluidin blue. The study of the cytopreparations was made by videomicroscope Axioscop 20, MC80, trinocular, Carl Zeiss.

\section{MLRs quantification}

A macrophage-lymphocytic rosette (MLR) was considered to be formed when a monocyte- macrophage was surrounded by three or more lymphocytes. The number of rosettes (MLRs) was expressed as the percentage of the number of macrophages that formed rosettes in a given cytopreparation by counting 100 macrophages.

\section{Statistical analysis}

The data was presented as the mean value \pm SD $(n=10)$ and represented at least three independent experiments from independent donors. ${ }^{* * *} p<0,001$ by paired Student's $t$-test using Infostat software. In MLR assay the data was presented as the mean value \pm SD $(n=3){ }^{* *} p<0,01$ by paired Student's $t$-test using Infostat software.

\section{Results}

\section{Generating NETs}

Cultured cells were stimulated to form NETs with LPS or OVA (Figures 1, 2 and 3). Presence of NETs was clearly showed by immunofluorescence staining of DNA with DAPI and one NETosis specific marker, the NE in LPS assays at 120 minutes (Figure 2B and $2 \mathrm{C}$ ). NETs visualize as blue diffuse staining of DAPI (DNA) or chromatin distribution in numerous fibrils, or "spray" aspect, a phenomenon that externalizes the content of the cell, in all tests of stimulation with LPS (Figure 1A and 1C) at 30 minutes, at 120 minutes (Figure 2B and 2C) and 24 hours (Figure 3A) or OVA at 30 minutes (Figure 1B) and 24 hours (Figure 3C). We found that stimulation with LPS or OVA increased the basal levels of NETs from neutrophils derived from healthy donors (Figure 1E and 3D). In OVA assay at $30 \mathrm{~min}$ fewer cells releasing NETs were observed compared with assay at 24 hours, the analysis obtained showed a significant difference ${ }^{* *} p<0.001$ (Figure $1 \mathrm{E}$ and $3 \mathrm{D}$ ).

\section{Colocalization of CD80 and CD86}

We performed the generation of NETs in autologous leukocytes cultures, stimulated with LPS or OVA and we marked CD80 and CD86 costimulatory molecules. The areas of red (CD86) and green (CD80) with immunofluorescence staining are identical, indicating colocalization in NETs. In LPS and OVA assays, CD80 was found to colocalize with CD86 in NETs released by stimulated PMNs at 30 minutes. These molecules are detected on the cell surface at 30 minutes (Figure $1 \mathrm{~A}$ and $1 \mathrm{~B}$ ). In the control unstimulated paired blood samples at $30 \mathrm{~min}$ CD80 was negative and CD86 was found stained in some cells (Figure 1D). In LPS assay at 30 minutes the immunofluorescence staining showed NE granular appearance and CD86 was found in some cells (Figure 2A).

In LPS assay at 120 minutes CD80 (green) colocalizes with NE (red) in NETs (Figure 2B) and CD86 (red) colocalizes with NE (green) in NETs (Figure 2C). In the control unstimulated samples at $120 \mathrm{~min}$ of culture, NE (green) CD86 (red) (Figure 2E) and NE (red) CD80 (green) (Figure 2D and 2F) were found stained inside cells. We performed IF to CD80 and CD86 in samples from MLRs assay, see next section, and was found colocalization in NETs.

In LPS and OVA assays, CD80 was found to colocalize with CD86 in NETs released by stimulated PMNs at 24 hours (Figure 3A and 3C). In the control unstimulated samples at 24 hours CD80 and CD86 were negative. In OVA assay, fewer cells with CD80 and CD86 in NETs were observed compared with LPS assays, the analysis obtained showed a significant difference ${ }^{\star * *} p<0.001$ (Figures $1 \mathrm{~F}$ and $3 \mathrm{E})$. 

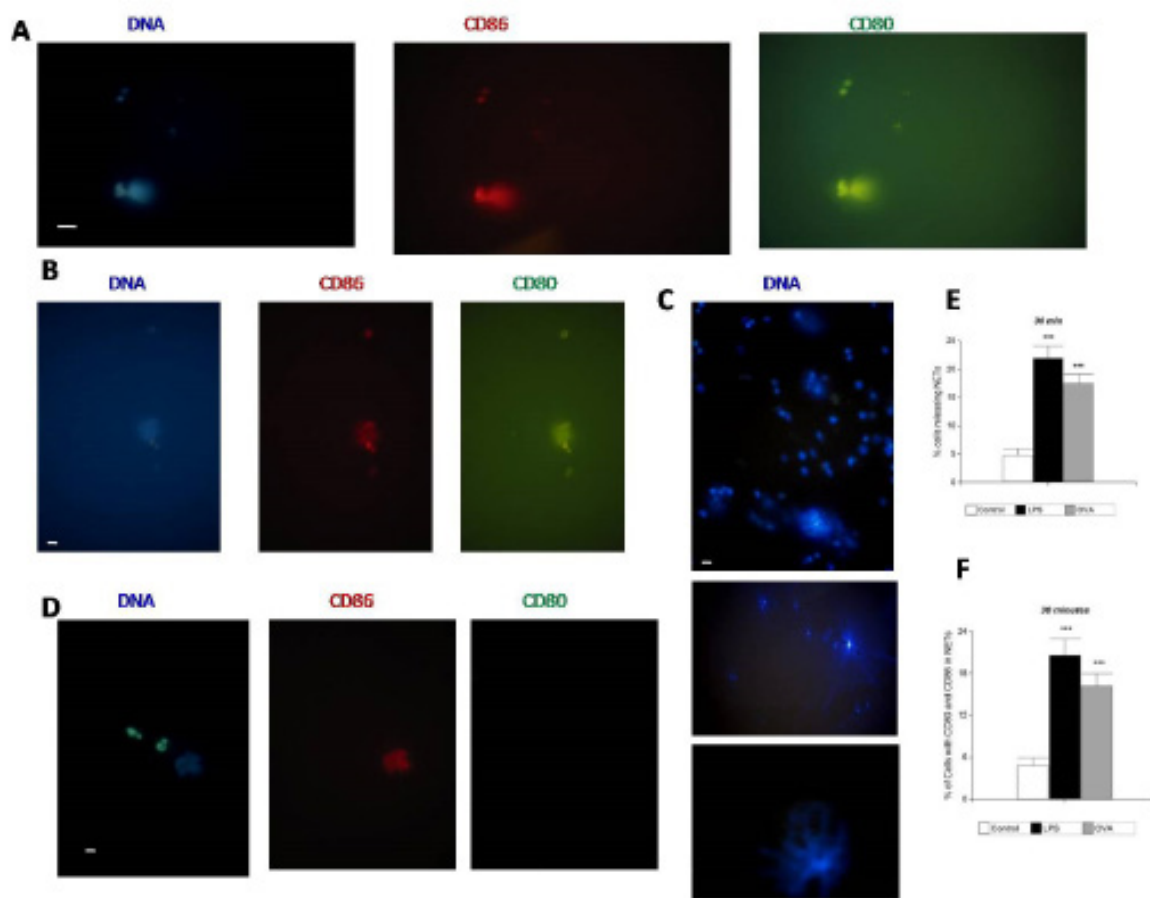

$\mathbf{F}$

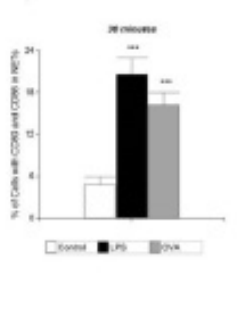

Figure 1: CD80 and CD86 colocalized in NETs after stimulation for 30 minutes; Immunofluorescence analysis, representative pictures from three independent experiments from independent donors; (A) Composite representative pictures of fluorescence microscopy showing CD80 (green) and CD86 (red). Pictures obtained after stimulation with LPS for 30 minutes. Scale bar represents $10 \mu \mathrm{m}$; (B) Pictures obtained after stimulation with OVA for 30 minutes showing CD80 (green) and CD86 (red) colocalized in NETs. Scale bar represents $10 \mu \mathrm{m}$; (C) Representative pictures obtained after stimulation with LPS for 30 minutes, DNA stained with DAPI (blue). Scale bar represents $10 \mu \mathrm{m}$; (D) Representative picture obtained without stimulation (control paired blood samples). DNA stained with DAPI, CD80 (green) and CD86 (red). Scale bar represents $10 \mu \mathrm{m}$; (E). Cells with stimulation with LPS or OVA for 30 minutes. The released NETs were visualized by immunofluorescence microscopy and the percentage of cells releasing NETs was calculated as the average of six fields (400x) normalized to the total number of cells. The data was presented as the mean value \pm SD of $n=10$ and represented at least three independent experiments from independent donors. ${ }^{* \star *} p<0,001$ by paired Student's $t$-test; (F) Cells with stimulation with LPS or OVA for 30 minutes. The released NETs were visualized by immunofluorescence microscopy and the percentage of cells releasing NETs with CD80 and CD86 colocalized in NETs was calculated as the average of six fields (400x) normalized to the total number of cells. The data was presented as the mean value $\pm \mathrm{SD}$ of $n=10$ and represented at least three independent experiments from independent donors. ${ }^{* *} p<0,001$ by paired Student's $t$-test

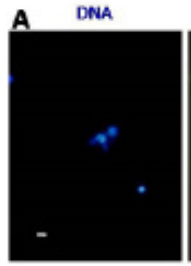

NI:

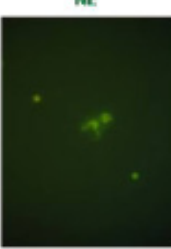

C DNA

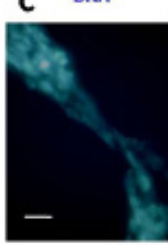

E

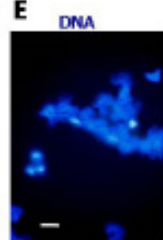

NE

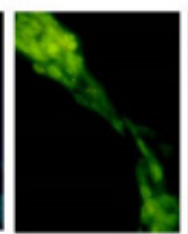

NE

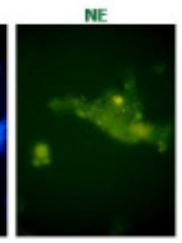

CD86

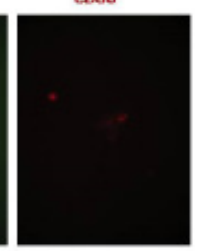

CD86

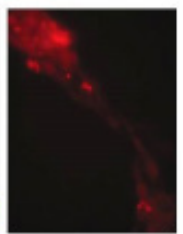

Co86

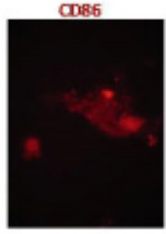

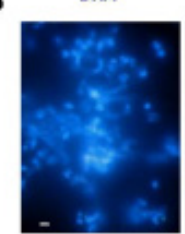

DNA

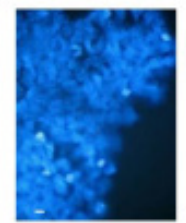

F DNA

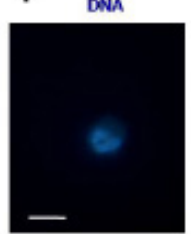

CD80

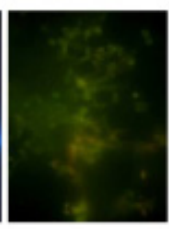

Cose

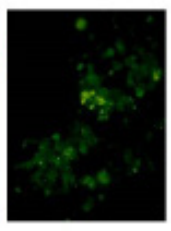

coso

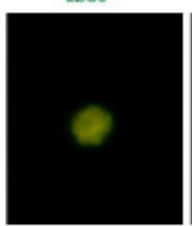

NE

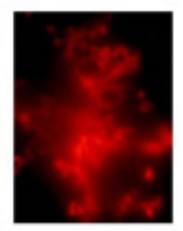

NE

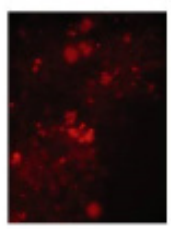

NE

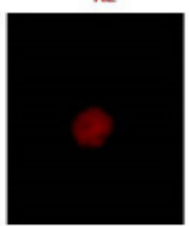

Figure 2: Immunofluorescence analysis of NE, CD80 and CD86. Representative pictures from three independent experiments from independent donors; (A) Pictures obtained after stimulation with LPS for 30 minutes. Scale bar represents $10 \mu \mathrm{m}$. DNA stained with DAPI (blue), CD86 (red) and NE (green); (B) Pictures obtained after stimulation with LPS for 120 minutes. Scale bar represents $10 \mu \mathrm{m}$. DNA stained with DAPI (blue), CD80 (green) and NE (red) colocalized in NETs; (C) Pictures obtained after stimulation with LPS for 120 minutes showing NE (green) and CD86 (red) colocalized in NETs. Scale bar represents $10 \mu$ m; (D) Representative picture obtained without stimulation (control paired blood samples) at 120 min of culture time. DNA stained with DAPI, CD80 (green) and NE (red). Scale bar represents $10 \mu \mathrm{m}$; (E) Representative picture obtained without stimulation (control paired blood samples) at 120 min of culture time. DNA stained with DAPI, NE (green) and CD86 (red). Scale bar represents $10 \mu \mathrm{m}$; (F) Representative picture of PMN obtained without stimulation (control paired blood samples) at $120 \mathrm{~min}$ of culture time. DNA stained with DAPI, CD80 (green) and NE (red). Scale bar represents $10 \mu \mathrm{m}$ 


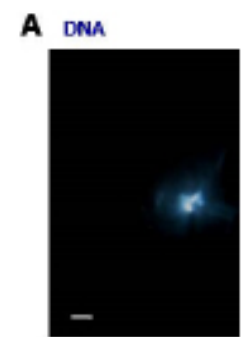

C
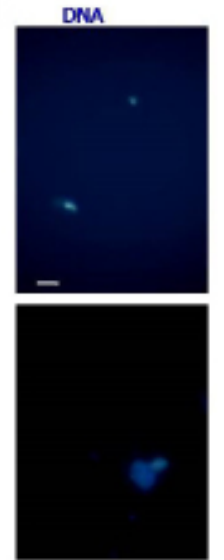

CD86

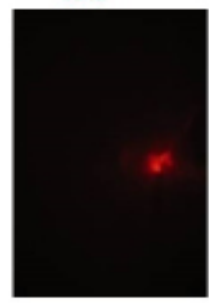

CD8Б
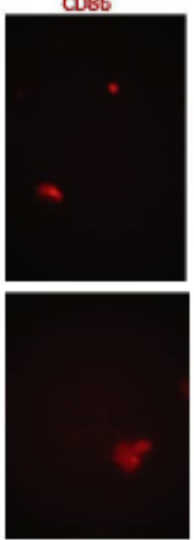

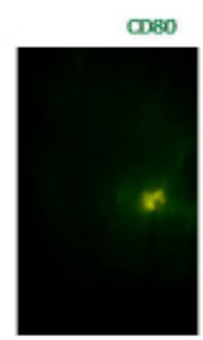

CDEO

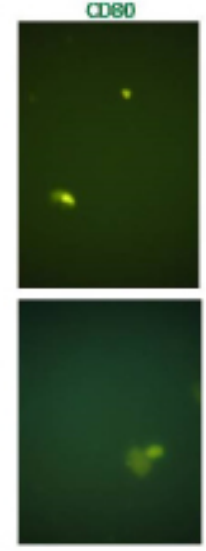

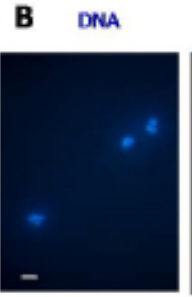

cos6

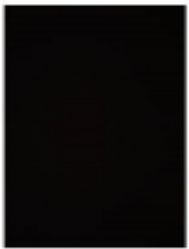

ma

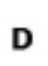

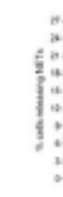

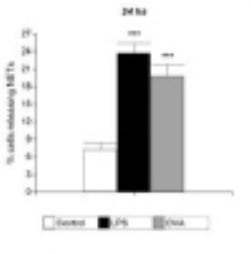

E

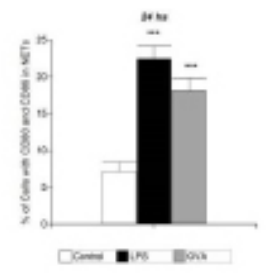

coso

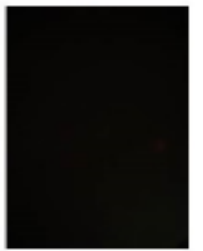

.

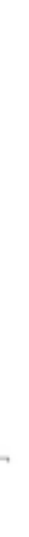

Figure 3: CD80 and CD86 colocalized in NETs after stimulation for 24 hours. Immunofluorescence analysis, representative pictures from three independent experiments from independent donors; (A) Composite representative pictures of fluorescence microscopy showing CD80 and CD86 colocalized in NETs. DNA stained with DAPI (blue), CD80 (green) and CD86 (red). Pictures obtained after stimulation with LPS for 24 hours. Scale bar represents $10 \mu$ m; (B) Representative picture obtained without stimulation (control paired blood samples). DNA stained with DAPI, CD80 (green) and CD86 (red). Scale bar represents $10 \mu$ m; (C) Composite representative pictures obtained after stimulation with OVA for 24 hours showing CD80 (green) and CD86 (red) colocalized in NETs. Scale bar represents $10 \mu \mathrm{m}$; (D) Cells with stimulation with LPS or OVA for 24 hours. The released NETs were visualized by immunofluorescence microscopy and the percentage of cells releasing NETs was calculated as the average of six fields (400x) normalized to the total number of cells. The data was presented as the mean value \pm SD of $n=10$ and represented at least three independent experiments from independent donors. ${ }^{\star * \star} p<0,001$ by paired Student's $t$-test; (E) Cells with stimulation with LPS or OVA for 24 hours. The released NETs were visualized by immunofluorescence microscopy and the percentage of cells releasing NETs with CD80 and CD86 colocalized in NETs was calculated as the average of six fields (400x) normalized to the total number of cells. The data was presented as the mean value $\pm \mathrm{SD}$ of $n=10$ and represented at least three independent experiments from independent donors. ${ }^{* * *} p<0,001$ by paired Student's $t$-test

\section{Generating MLRs with OVA stimulation}

Autologous leukocytes cultures were stimulated by addition of OVA heterologous antigen. Samples of these cultures were harvested at 24 hours and cytopreparations were made from cell pellets as described above in the MLR assay section. We observed the occurrence of MLRs in cell culture samples pelleted at 200g and in the same samples we found CD80 and CD86 colocalized in NETs by IF (Figures 4A, 4C and 4D). A higher number of MLRs was formed on the cytopreparations, as compared with controls without OVA. The analysis obtained showed a significant difference ${ }^{\star *} p<0.01$ (Figure $4 \mathrm{~B}$ ). We generated a higher number of MLRs with OVA stimulation in samples with presence of CD80 and CD86 in NETs.

\section{Discussion}

The mechanism of NETs' formation implies nuclear membranes and granules disintegrates. The components of nucleus and cytoplasm mix and the plasmatic membrane breaks and ejects the interior of the cell to the extracellular space, thus forming NETs [5].

It is described in the bibliography that B7 molecules can be pre-formed in cytoplasmic reservoirs. The granular components are part of the NETs, so we hypothesize that costimulatory molecules may also be found forming part thereof.

In this study, we perform assays to generate NETs in human autologous leukocytes cultures, and we marked CD80 and CD86 costimulatory molecules. The incubation with 5\% blocking serum albumin in PBS to prevent non-specific staining was performed.

Sandilands et al. describe cytoplasmic reservoirs of molecules B7: CD80 mainly within secretory vesicles and CD86 within secondary, azurophilic granules and secretory vesicles [29]. In this work we found costimulatory molecules inside the cells in samples of unstimulated controls: at 30 minutes CD86 was positive, at 120 minutes CD86 and CD80 were positive but at 24 hours CD80 and CD86 were negative (Figures 1, 2 and 3). At 30 minutes CD86 was found stained in some cells in cell surface (Figure 1D). At 120 minutes CD86 was found in cytoplasm (Figure 2E) in control unstimulated paired blood samples and CD80 was found in cytoplasm (Figure 2D and 2F) in control unstimulated paired blood samples. The samples permeabilized (Figure 2) show stain inside the cells in cytoplasmic reservoirs of molecules CD80 and CD86 [29]. 
A

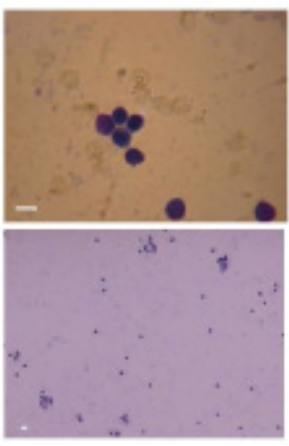

C

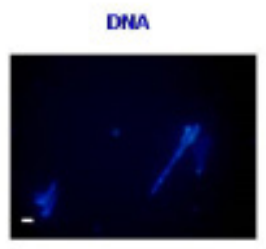

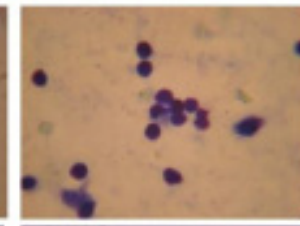
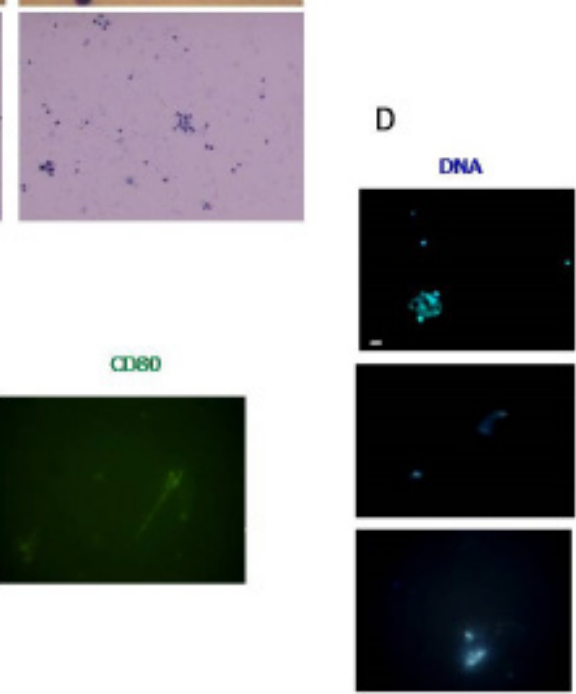

B

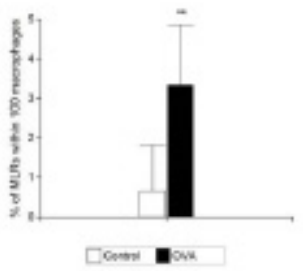

coso

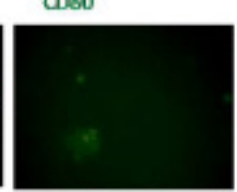

CDe66

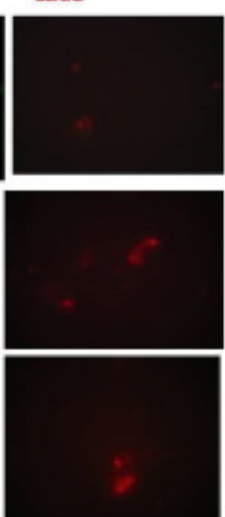

Figure 4: CD80 and CD86 colocalized in NETs and Monocyte-macrophage Lymphocytic Rosettes (MLRs) assay with OVA addition; (A) Composite representative pictures of light microscopy showing MLRs in samples of autologous leukocytes cultures after stimulation with heterologous antigen OVA for $24 \mathrm{~h}$. Cytopreparations were stained with May Grünwald Giemsa or Toluidin blue. Scale bar represents $10 \mu \mathrm{m}$; (B) Graph of Monocyte-macrophage Lymphocytic Rosettes (MLRs) assay with stimulation with heterologous antigen OVA for $24 \mathrm{~h}$. A macrophage-lymphocytic rosette (MLR) was considered to be formed when a monocyte- macrophage was surrounded by three or more lymphocytes. The number of rosettes (MLRs) was expressed as the percentage of the number of macrophages that formed rosettes in a given cytopreparation by counting 100 macrophages. The data was presented as the mean value \pm SD $(n=3){ }^{* *} p<0,01$ by paired Student's $t$-test using Infostat software; (C) Composite representative pictures of fluorescence microscopy showing CD80 (green), DNA stained with DAPI (blue) in samples of autologous leukocytes cultures after stimulation with heterologous antigen OVA for $24 \mathrm{~h}$ and MLRs assay performed. Scale bar represents $10 \mu \mathrm{m}$; (D) Composite representative pictures of fluorescence microscopy showing CD80 (green) and CD86 (red), DNA stained with DAPI (blue) in samples of autologous leukocytes cultures after stimulation with heterologous antigen OVA for $24 \mathrm{~h}$ and MLRs assay performed. Scale bar represents $10 \mu \mathrm{m}$

These findings are consistent with temporal expression pattern of molecules CD80 and CD86. It has been established that the cross linking of Mac 1 (CD11b+CD18) results in the translocation of CD80, CD86 and HLA-DR on the surface of normal human peripheral blood PMNs. This represents one way of PMN activation. There are many well documented neutrophils activators. Between the activators, LPS is one of the most recognized. LPS stimulates the release of secretory vesicles [29] and NETs release [11]. In our present study released NETs were confirmed by immunofluorescence staining of DNA with DAPI and one NETosis specific marker like NE. NETs are fragile structures [5]. The mixture of cytoplasm and nucleoplasm gets expelled to form NETs [5]. NETs visualize as blue diffuse staining of DAPI (DNA) or chromatin distribution in numerous fibrils, or "spray" aspect in all tests of stimulation with LPS (Figure 1A, 1C, 2B, 2C, and 3A) or OVA (Figure 1B and 3C). In LPS assay at 30 minutes the immunofluorescence staining showed NE granular appearance and CD86 was found in some cells (Figure 2A). At 30 min NE localizes inside azurophilic granules. It has been described that NE localized to the nucleus 60 min after stimulation, accompanied by reduced granular staining [10]. NE migrates from the azurophilic granules to the nucleus and partially degrades histones promoting chromatin decondensation in NET formation process. In Figure 2B and 2C we showed results with NE at 120 min and NE colocalized with chromatin in agree with bibliography [10]. Colocalized molecules CD80 and CD86 with specific antibodies were observed. This finding would be possible because after the activation, neutrophils release NETs comprising decondensed chromatin associated with granular and cytosolic proteins. In stimulated samples CD80 and CD86 were detected on the cell surface and colocalized in NETs at 30 minutes (Figure 1A and 1B), 120 minutes (Figure 2B and 2C) and 24 hours (Figure 3A and 3C). The results in the control unstimulated paired blood samples at 30 minutes showed that CD80 was negative and CD86 was detected in some cells (Figure 1D) may be monocytic, in agreement with Smyth et al. (2004). In LPS assay at 120 minutes, CD86 and CD80 were found in control samples (Figure 2D and 2F).

Other researchers observed the presence of neutrophil-bearing antigen in lymphoid organs of immune mice. Although it has been generally accepted that neutrophils are not involved in adaptive responses, the uptake of OVA antigen by neutrophils was observed. These neutrophils principally secrete TNF alfa and authors conclude this would let them participate as immunomodulatory cells [30]. NETs were induced by immuno complexes OVA:anti-OVA [31] and in other granulocytes, formation of extracellular traps induced by OVA were described [24]. 
Journal of Immunology and Infectious Diseases

In the present study, we used whole blood to avoid spontaneous activation of neutrophils [29]. Having used whole blood, there are chances that other granulocytes produce extracellular traps (ET) too [24], but in the present work, we can attribute the generation of ETs to neutrophils because they represent $60-70 \%$ of the circulating leukocytes (lymphocytes represent $20-30 \%$ and them have not been described like ET generating cells) and because we used NE as a specific marker of NETs [24]. NETs have an "unique composition: chromatin tightly linked to neutrophil proteins such as NE, MPO, or calgranulin, and this definition excludes chromatin released by other forms of cell death" [5].

We observed NETs generation after stimulation with LPS or OVA at different times but in OVA assay we observed fewer cells with CD80 and CD86 in NETs $(p<0.001)$. The results of OVA assays would be possibly from OVA alone and/or OVA-anti OVA immune complexes due to the possible existence of specific anti-OVA antibodies in serum of donors. Controls not stimulated with LPS or OVA, showed little or no expression of CD80 and CD86.

When we started this work, we observed in a first time $(30 \mathrm{~min})$, the presence of costimulatory molecules in NETs. As it is well known, PMN have a short life; however, under certain conditions of stimulation they can live more time. After 24 hours of culture we observed the release of NETs too, so we decided to detect if CD80 and CD86 could be present in NETs.

The importance of this finding resides in neutrophils functions, the possibility of acquire competence to be APC and playing a role in immunomodulation. The presence of CD80 and CD86 molecules in NETs would let them influence the cell environment, activation of B7-1/B7-2:CD28/CTLA-4 pathway can result in stimulation or inhibition of other cells, such as activated T cells or $\mathrm{T}$ reg cells. This finding could have relevance for a break in immune tolerance mediated by NETs. The CD80 and CD86 molecules have characteristics of key that opens different doors, in relation to receptor CD28 or CTLA4, therefore the effects will be different, opposite and the responses may be stimulatory, inhibitory or anergic, considering the complexity of diverse populations of $\mathrm{T}$ cells, naives and effectors. Accordingly these cells can modulate the immune response switching phenotypes and exert functions to interplay with other immune cells resulting in proinflammatory or antiinflammatory profiles [32]. Neutrophils demonstrate they are versatile cells, capable of modulating adaptive immune responses, and not simply limiting their role to phagocytosis, as was described at the beginning.

An experiment with T cells was performed to assess potential functional relevance of CD80 and CD86 colocalized in NETs. We generated immunological synapses in MLRs. The MLRs are a phenomenon of antigen processing and presentation [28]. It has been described that lymphocytes forming the rosettes MLRs are T-CD4 cells [26]. In a previous study we showed that addition of heterologous antigen stimulated MLRs in cultures of purified mononuclear cells [28]. In this work we performed a MLRs assay with addition of OVA (antigen heterologous) in total autologous leukocyte cultures and a higher number of MLRs was formed. In the same samples we found CD80 and CD86 colocalized in NETs by IF. Control experiments were leukocyte cultures of the same donors without OVA. In previous works we hypothetized that the presence of autologous neutrophils or of the materials derived from them during culturing, could provide a microenvironment that enhances the formation of MLRs because highest number of MLR occurred in total leukocyte samples, whereas only a few of them appeared in the mononuclear cell samples [26]. Here OVA was the heterologous antigen processed and presented besides the own antigens derived from senescent neutrophils considering the natural (spontaneous) death of neutrophils in this time of culture. In this study the presence of costimulatory molecules in NETs is not a requisite for the MLRs occurrence but it would be relevant in the presence of the higher number of MLRs because antigen processing and presentation are involved in MLR phenomenon like it was demonstrated experimentally in previous work [27]. CD80 and CD86 present in NETs may be responsible in part for modulating T response in MLRs, but the increment in MLRs after OVA stimulus may be a consequence of central monocyte-macrophage which express costimulatory molecules against heterologous stimulus besides NETs or not netotic PMN. This finding could have relevance for a break in immune tolerance mediated by NETs. This possibility requires elucidation by further works to demonstrate the impact of NETs on functions of T cells.

\section{Conclusion}

In summary, we found CD80 and CD86 colocalized in NETs. The expression of costimulatory molecules in NETs would allow PMNs to play function as APCs and modulatory functions of various subpopulations of T cells. It needs to be probed with assays to evaluation of $\mathrm{T}$ cell responses. It is estimated of great interest the further study of the neutrophils phenotypes in interactions with other cell types. Experiments are currently underway to test it. This is the first communication of CD80 and CD86 costimulatory molecules in NETs and we consider it, a very significant finding for the immunological community.

\section{Acknowledgments}

We thank the Blood Bank, Institute of Hematology and Hemotherapy, National University of Cordoba for donation of blood samples. We thank Dr Cristina Maldonado for providing the primary antibody anti-NE and secondary antibodies. We thank Professor Ana M. Granata for language editing of the work. We are also grateful to Reviewers whose comments helped us make amendments to our manuscript. We thank National University of Cordoba, Argentina, Science and Technology Secretary for supported this work. 


\section{Funding Information}

This work was supported by grants from the National University of Cordoba, Argentina, Science and Technology Secretary (SECYT, UNC R 379/15).

\section{References}

1. Mantovani A, Cassatella MA, Costantini C, Jaillon S (2011) Neutrophils in the activation and regulation of innate and adaptive immunity. Nat Rev immunol 11: 519-31.

2. Phillipson M, Kubes P (2011) The neutrophil in vascular inflammation. Nat Med 17: 1381-90.

3. Brinkmann V, Reichard U, Goosmann C, Fauler B, Uhlemann Y, et al. (2004) Neutrophil extracelular traps kill bacteria. Science 303: 1532-5.

4. Kolaczhowska E, Kubes P (2013) Neutrophil recruitment and function in health and inflammation. Nat Rev Immunol 13: 159-75.

5. Brinkmann V, Zychlinsky A (2012) Neutrophil extracellular traps: Is immunity the second function of chromatin? J Cell Biol 198: 773-83.

6. Schorn C, Janko C, Krenn V, Zhao Y, Munoz LE, et al. (2012) Bonding the foe - NETting neutrophils immobilize the pro-inflammatory monosodium urate crystals. Front Immunol 3: 10.3389/fimmu.2012.00376.

7. Tillack K, Breiden P, Martin R, Sospedra M (2012) T Lymphocyte priming by neutrophil extracellular traps links innate and adaptive immune responses. J Immunol 188: 3150-9.

8. Barrientos L, Marin-Esteban V, de Chaisemartin L, Le-Moal VL, Sandré C, et al. (2013) An improved strategy to recover large fragments of functional human neutrophil extracellular traps. Front Inmunol 4: 10.3389/fimmu.2013.00166.

9. Berger-Achituv S, Brinkmann V, Abed UA, Kühn LI, Ben-Ezra J, et al. (2013) A proposed role for neutrophil extracelular traps in cancer immunoediting. Front Immunol 4: 10.3389/fimmu.2013.00048.

10. Papayannopoulos V, Metzler KD, Hakkim A, Zychlinsky A (2010) NE and myeloperoxidase regulate the formation of neutrophil extracellular traps. J Cell Biol 191: 677-91.

11. Branzk N, Papayannopoulos V (2013) Molecular mechanisms regulating NETosis in infection and disease. Semin Immunopathol 35: 513-30.

12. Kumar V, Sharma A (2010) Neutrophils: cinderella of innate immune system. Int Immunopharmacol 10: 1325-34.

13. Yipp BG, Kubes P (2013) NETosis: how vital is it? Blood 122: 2784-94.

14. Smyth CM, Logan G, Boadle R, Rowe PB, Smythe JA, et al. (2005) Differential subcellular localization of CD86 in human PBMC-derived macrophages and DCs, and ultrastructural characterization by immuno-electron microscopy. Int Immunol 17: 123-32.

15. Sandilands GP, Ahmed Z, Perry N, Davison M, Lupton A, et al. (2005) Cross-linking of neutrophil CD11b results in rapid cell surface expression of molecules required for antigen presentation and T-cell activation. Immunology 114: 354-68.

16. Lafferty KJ, Andrus L, Prowse SJ (1980) Role of lymphokine and antigen in the control of specific T cell responses. Immunol Rev 51: 279-314.

17. Sharpe AH (2009) Mechanisms of Costimulation. Immunol Rev 229: 5-11.

18. Chen L, Flies DB (2013) Molecular mechanisms of T cell co-stimulation and co-inhibition. Nat Rev Immunol 13: 227-42.

19. June CH, Ledbetter JA, Linsley PS, Thompson CB (1990) Role of the CD28 receptor in T-cell activation. Immunol Today 11: 211-6.

20. Brunet JF, Denizot F, Luciani MF, Roux-Dosseto M, Suzan M, et al. (1987) A new member of the immunoglobulin superfamily--CTLA-4. Nature 328: 267-70.

21. Hou TZ, Qureshi OS, Wang CJ, Baker J, Young SP, et al. (2015) A transendocytosis model of CTLA-4 function predicts its suppressive behavior on regulatory T cells. J Immunol 194: 2148-59.

22. Linsley PS, Brady W, Urnes M, Grosmaire LS, Damle NK, et al. (1991) CTLA-4 is a second receptor for the B cell activation antigen B7. J Exp Med 174: 561-9.

23. Qureshi OS, Zheng Y, Nakamura K, Attridge K, Manzotti C, et al. (2011) Trans-endocytosis of CD80 and CD86: a molecular basis for the cell extrinsic function of CTLA-4. Science 332: 600-3.

24. Cunha AA, Porto BN, Nuñez NK, Souza RG, Vargas MH, et al. (2014) Extracellular DNA traps in bronchoalveolar fluid from a murine eosinophilic pulmonary response. Allergy 69: 1696-700.

25. Cabral HR, Novak I (1992) Spontaneous formation of rosettes by autologous human monocyte- macrophages and lymphocytes in cell cultures. Rev Fac Cien Med Univ Nac Cordoba 50: 25-6.

26. Cabral HR, Novak IT (1999) Autologous rosette formation by human blood monocyte-derived macrophages and lymphocytes. Am J Hematol 60: 285-8.

27. Novak IT, Cabral HR (2008) Rossette formation by macrophages with adhered T lymphocytes is precluded by inhibitors of antigen processing and presentation. Biocell 32: 169-74.

28. Novak I and Cabral H (2009) Immunological Synapses Formation: Rosettes between Human Autologous Cultured Monocyte-Macrophages and Lymphocytes, Proceedings of 2nd European Congress of Immunology. ECI, MEDIMOND International Proceedings, L913C0012, p.117-121

29. Sandilands GP, McCrae J, Hill K, Perry M, Baxter D (2006) Major histocompatibility complex class II (DR) antigen and costimulatory molecules on in vitro and in vivo activated human polymorphonuclear neutrophils. Immunology 119: 562-71.

30. Maletto BA, Ropolo AS, Alignani DO, Liscovsky MV, Ranocchia RP, et al. (2006) Presence of neutrophil-bearing antigen in lymphoid organs of immune mice. Blood 108: 3094-102.

31. Yu Y, Koehn CD, Yue Y, Li S, Thiele GM, et al. (2015) Celastrol Inhibits Inflammatory Stimuli-Induced Neutrophil Extracellular Trap Formation. Curr Mol Med 15: 401-10.

32. Perobelli SM, Galvani RG, Goncalves-Silva T, Xavier CR, Nobrega A, et al. (2015) Plasticity of neutrophils reveals modulatory capacity. Braz J Med Biol Res 48: 665-75. 


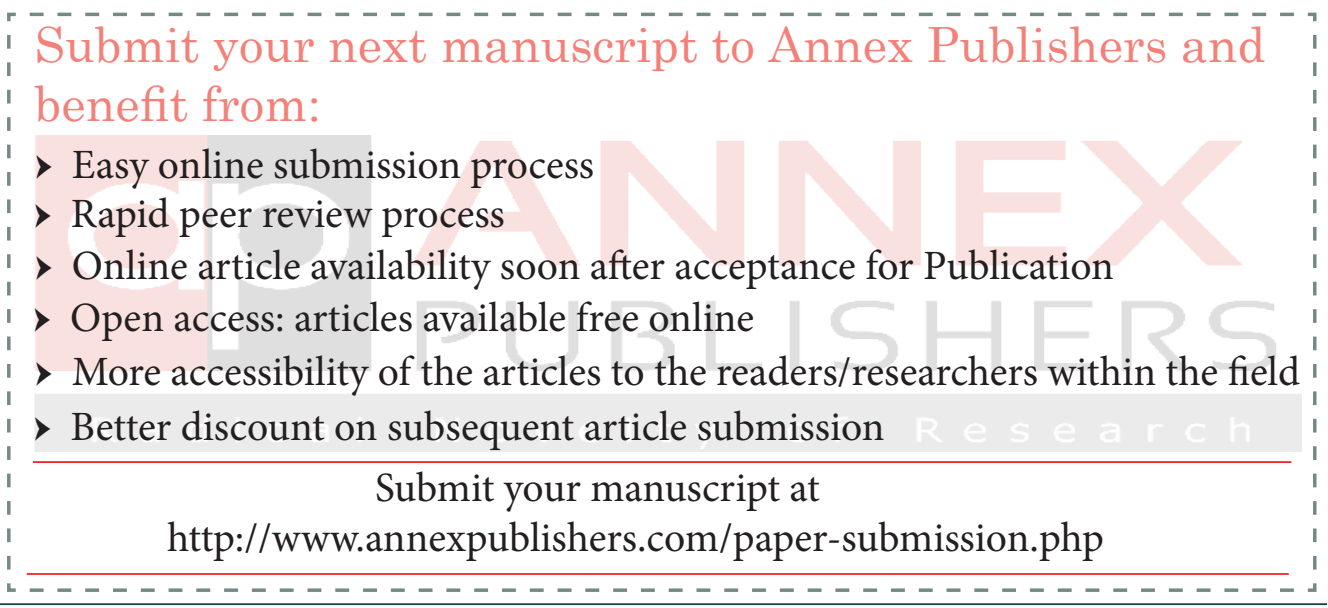

